\title{
ERRATUM
}

\section{Erratum to: Who Shot Ya? How Emergency Departments Can Collect Reliable Police Shooting Data}

\author{
Joseph B. Richardson
}

Erratum to: J Urban Health

10.1007/s11524-015-0008-7

Please note that there were two authors missing from the author line of this article as originally published.

Their names and affiliations are:

Christopher St. Vil, Assistant Professor University at Buffalo, SUNY

School of Social Work

685 Baldy Hall

Buffalo, NY 14260

cstvil@buffalo.edu

Carnell Cooper

Associate Professor of Surgery

University of MD School of Medicine

Attending, RA Cowley Shock Trauma Center

Chief Medical Officer, Prince George's Hospital Center

email: ccooper@umm.edu; carnell.cooper@dimensionshealth.org

Richardson is with the Department of African-American Studies Violence Intervention Research Project, Prince George's Hospital Trauma Center, College Park, MD, USA.

Correspondence: Joseph B. Richardson, Jr., Department of African-American Studies Violence Intervention Research Project, Prince George's Hospital Trauma Center, College Park, MD, USA. (Email: jrichar5@umd.edu)

The online version of the original article can be found at http://dx.doi.org/10.1007/s11524-0150008-7. 